\title{
Stability of rotating spherical stellar systems
}

\author{
A. $\mathrm{Meza}^{\star, \star \star}$ \\ Departamento de Astronomía y Astrofísica, Pontificia Universidad Católica de Chile, Casilla 306-22, Santiago, Chile
}

Received 13 August 2001 / Accepted 30 August 2002

\begin{abstract}
The stability of rotating isotropic spherical stellar systems is investigated by using $N$-body simulations. Four spherical models with realistic density profiles are studied: one of them fits the luminosity profile of globular clusters, while the remaining three models provide good approximations to the surface brightness of elliptical galaxies. The phase-space distribution function $f(E)$ of each one of these non-rotating models satisfies the sufficient condition for stability $\mathrm{d} f / \mathrm{d} E<0$. Different amounts of rotation are introduced in these models by changing the sign of the $z$-component of the angular momentum for a given fraction of the particles. Numerical simulations show that all these rotating models are stable to both radial and non-radial perturbations, irrespective of their degree of rotation. These results suggest that rotating isotropic spherical models with realistic density profiles might generally be stable. Furthermore, they show that spherical stellar systems can rotate very rapidly without becoming oblate.
\end{abstract}

Key words. celestial mechanics - stellar dynamics - galaxies: kinematics and dynamics - instabilities methods: $N$-body simulations

\section{Introduction}

Dynamical instabilities in spherically symmetric stellar systems have been investigated for more than four decades. In a seminal work, Antonov $(1960,1962)$ used a variational principle to demonstrate that non-rotating spherical models with a phase-space distribution function $f$ depending only on the energy $E$ are stable to non-radial perturbations if $\mathrm{d} f / \mathrm{d} E<0$. Subsequent works showed that this condition is also a sufficient condition for stability to radial perturbations (Dorémus et al. 1971; Sygnet et al. 1984; Kandrup \& Sygnet 1985). In general, non-rotating spherical stellar systems are described by distribution functions $f$ that depend on both the energy $E$ and the magnitude of the angular momentum $L$. In such systems only the stability to radial modes can be tested by using the sufficient condition $\partial f / \partial E<0$ (Dorémus \& Feix 1973; Dejonghe \& Merritt 1988). For this reason, numerical simulations have been an indispensable tool to investigate the stability of anisotropic spherical models.

Several classes of instabilities have been discovered in nonrotating spherical models with anisotropic velocity distributions (e.g., Hénon 1973; Merritt \& Aguilar 1985; Barnes et al. 1986; see Merritt 1999 for a recent review). For example, models dominated by stars on radial or eccentric orbits can be unstable to forming a triaxial bar (Polyachenko 1981; Merritt \& Aguilar 1985; Meza \& Zamorano 1997), while models

$\star$ e-mail: ameza@uvic.ca

$\star \star$ Present Address: Department of Physics and Astronomy, University of Victoria, Victoria BC, V8P 1A1, Canada. composed mainly of stars on circular orbits can exhibit quadrupole-mode oscillations (Barnes et al. 1986).

In contrast to non-rotating spherical models, comparatively little work has been done to investigate the stability of rotating spherical stellar systems. Miller \& Smith (1980) employed several methods to introduce rotation in a spherical $n=3$ polytrope with isotropic velocity distribution. In all cases, they found that the addition of rotation does not affect the stability of their models; indeed, they found that even rapidly rotating systems remain spherically symmetric. More recently, Alimi et al. (1999) showed that rotating isotropic spherical $n=4$ polytropes, which were made to rotate by changing the sign of the $z$-component of the angular momentum for a fraction of the particles, were dynamically stable.

On the other hand, Allen et al. (1992) reported the existence of a "tumbling bar instability" in a set of rotating spherical models with different degrees of velocity anisotropy, ranging from completely circular to entirely radial (see also Papaloizou et al. 1991; Palmer 1994a, 1994b). In particular, they suggested that the introduction of a small amount of rotation in the isotropic spherical $n=2$ polytrope induces a bar instability in this otherwise stable system. However, Sellwood \& Valluri (1997) using the same files of initial conditions but a different $N$-body code, showed that these models are actually stable; the instability appears do not exist. They suggested that the evolution observed by Allen et al. (1992) was, probably, caused by an improper treatment of variable time steps in their $N$-body code.

Most of the previous results have been obtained for rotating spherical models with somewhat unrealistic properties. Indeed, 
most of these works have employed spherical polytropes with finite radius (e.g., Alimi et al. 1999) or models with unrealistic velocity distributions (e.g., Allen et al. 1992). Therefore, they cannot be considered as general. To investigate the influence of rotation on the stability of spherical stellar systems, it is necessary to consider models with more realistic density profiles and velocity distributions. In this paper, the results of a series of $N$-body simulations for four of such models are presented. These isotropic spherical models are: the Plummer (1911) model, the Hernquist (1990) model, the Jaffe (1983) model, and the $\gamma=0$ model (Dehnen 1993; Tremaine et al. 1994). Different degrees of rotation were introduced in these models by using the so-called Lynden-Bell's $(1960,1962)$ demon to reverse the sense of rotation along the $z$-axis of a given fraction of the particles. Numerical simulations show that all these rotating models are stable, regardless of their degree of rotation.

This paper is organized as follows. The method employed to introduce net rotation in these models and the $N$-body code used to follow their dynamical evolution are described in Sect. 2. The main results of the numerical simulations are summarized in Sect. 3. Finally, a brief discussion of the results is given in Sect. 4.

\section{Numerical simulations}

\subsection{Rotating models}

The spherical models studied in this paper are: the Plummer (1911) model,

$\rho=\frac{3}{4 \pi} \frac{M a^{2}}{\left(a^{2}+r^{2}\right)^{5 / 2}}$,

the Jaffe (1983) model,

$\rho=\frac{1}{4 \pi} \frac{M a}{r^{2}(a+r)^{2}}$,

the Hernquist (1990) model,

$\rho=\frac{1}{2 \pi} \frac{M a}{r(a+r)^{3}}$

and the $\gamma=0$ model (Dehnen 1993; Tremaine et al. 1994),

$\rho=\frac{3}{4 \pi} \frac{a M}{(a+r)^{4}}$.

In all these cases, $M$ is the total mass and $a$ is the scale radius. Hereafter, all quantities are expressed in units such that $M=a=G=1$, where $G$ is the gravitational constant. Some parameters for these models are summarized in Table 1, where $r_{\mathrm{h}}$ is the half-mass radius and $t_{\mathrm{h}}$ is the dynamical time evaluated at $r_{\mathrm{h}}$.

The Plummer model fits the light distribution of globular clusters (see e.g., Spitzer 1987), while the remaining three density profiles provide good approximations to the surface brightness of elliptical galaxies (see Dehnen 1993). Several intrinsic properties and projected quantities of these models can be obtained analytically, e.g., the velocity dispersions, the surface brightness profile and the mass distribution. In particular,
Table 1. Parameters for the models.

\begin{tabular}{lcrl}
\hline \hline Model & $r_{\mathrm{h}}$ & $t_{\mathrm{h}}$ & $\Delta t$ \\
\hline Plummer & 1.31 & 3.32 & 0.008 \\
Hernquist & 2.41 & 8.33 & 0.02 \\
Jaffe & 1.00 & 2.22 & 0.006 \\
$\gamma=0$ & 3.85 & 16.78 & 0.04 \\
\hline
\end{tabular}

the phase-space distribution function $f(E)$ can be obtained analytically by using the Eddington's inversion formula (see e.g., Binney \& Tremaine 1987). For all these non-rotating models, the distribution function satisfies the sufficient condition for stability $\mathrm{d} f / \mathrm{d} E<0$. Therefore, the models are stable to both radial and non-radial perturbations (Antonov 1962; Doremus et al. 1971; Sygnet et al. 1984; Kandrup \& Sygnet 1985).

Initial conditions for the simulations were derived from the density profile and the distribution function by using the following scheme. The radial coordinate of each particle is assigned by inverting the equation $M(r)=x$, where $M(r)$ is the total mass inside the radius $r$ and $x$ is a uniform random variable in the range $[0,1)$. Then, the coordinates of the position vector $\boldsymbol{x}$ are chosen at random from a sphere with radius $r$. The distribution function $f(E)$ and the gravitational potential $\Phi(r)$ evaluated at the particle position define the distribution of velocities, which is sampled by an acceptance-rejection technique to assign the modulus of the velocity $v$ of each particle (see e.g., Press et al. 1992). Finally, the coordinates of the velocity are chosen at random from a sphere with radius $v$.

The distribution function $f(E)$ does not depend on the sign of the $z$-component of the angular momentum $L_{z}$ (or equivalently on the sign of $v_{\phi}$ ), therefore the models have no net streaming. Different amounts of rotation can be introduced in these models by reversing the sense of rotation about some axis, here taken to be the $z$-axis, of a given fraction of the particles (Lynden-Bell 1960, 1962). In a non-rotating model, there are equal number of particles with $v_{\phi}$ going in opposite directions, while for a maximally streaming model the sign of $v_{\phi}$ is the same for all particles. All intermediate cases have varying fractions of particles with $v_{\phi}$ going in opposite directions. This scheme preserves the position and the norm of the velocity of each particle, then the systems are put in rotation without modifying their total kinetic and potential energy. Therefore, the resulting rotating models are also in dynamical equilibrium (see Lynden-Bell 1960).

This flipping rule introduces a discontinuity in the distribution function across $L_{z}=0$, which, in principle, can enhance the strength of the bar mode (Kalnajs 1977). However, as it is shown below, no signs of bar instabilities are observed in these rotating models. Therefore, no special procedure was necessary to taper this flipping rule when $\left|L_{z}\right|$ is small.

The degree of rotation in these models can be measured by the parameter (see e.g., Sellwood \& Valluri 1997)

$\eta=\frac{\sum_{i=1}^{N} L_{z_{i}}}{\sum_{i=1}^{N}\left|L_{z_{i}}\right|}$,

which varies from $\eta=0$ for a non-rotating model to $\eta=1$ for a model with all particles orbiting in the same sense around 
Table 2. Parameters for the rotating models.

\begin{tabular}{cc}
\hline \hline$\eta$ & $\mu$ \\
\hline 0 & 0.17 \\
0.2 & 0.20 \\
0.4 & 0.23 \\
0.6 & 0.27 \\
0.8 & 0.30 \\
1.0 & 0.33 \\
\hline
\end{tabular}

the $z$ axis. When $\eta=0.5$ the system has half the maximum possible total angular momentum; in this case, $75 \%$ of the particles are orbiting in the direct sense and the remaining $25 \%$ are retrograde.

Alternatively, the amount of rotation can also be given in terms of the following parameter (see Navarro \& White 1993)

$\mu=\frac{K_{\mathrm{rot}}}{K}$,

where $K$ is the total kinetic energy and $K_{\text {rot }}$ is the rotation kinetic energy defined by

$K_{\mathrm{rot}}=\frac{1}{2} \sum_{i=1}^{N} \frac{m_{i}\left(\boldsymbol{L}_{i} \cdot \hat{\boldsymbol{L}}_{\mathrm{tot}}\right)^{2}}{r_{i}^{2}-\left(\boldsymbol{r}_{i} \cdot \hat{\boldsymbol{L}}_{\mathrm{tot}}\right)^{2}}$.

In order to exclude counterrotating particles, the sum in Eq. (7) is carried out only over particles that satisfy the condition $\left(\boldsymbol{L}_{i} \cdot \hat{\boldsymbol{L}}_{\mathrm{tot}}\right)>0$, where $\hat{\boldsymbol{L}}_{\text {tot }}$ is a unit vector in the direction of the total angular momentum of the system, in this case, along the $z$-axis. Therefore, this parameter measures the kinetic energy in the rotational motion around the $z$-axis. It varies from $\mu=1 / 6$ for a non-rotating model to $\mu=1 / 3$, the maximum value allowed for the virial theorem, for a model with all particles rotating in the same sense around the $z$-axis. Table 2 summarizes the values of $\eta$ and the associated values of $\mu$ employed in the simulations.

Figure 1 shows the mean streaming velocity for Plummer models with different degrees of rotation. To measure this quantity, the systems were first projected along the $y$-axis and, then, a slit was placed along the projected $x$-axis. The slit width was set at $r_{\mathrm{h}}$, the half-mass radius, and the bin size along the projected axis was varied such that each bin contains the same number of particles $\left(n_{\text {bin }}=1000\right)$. In all the rotating models, the velocity curve reaches the maximun at $R \lesssim r_{\mathrm{h}}$ and then decreases slowly (models with $\eta \gtrsim 0.6$ ) or remains practically flat (models with $0<\eta \lesssim 0.6$ ). As expected, the mean streaming velocity of the non-rotating model $(\eta=0)$ is nearly zero; the observed fluctuations reflect the discreteness of the models. The velocity curves for the other models are very similar.

\subsection{N-body code}

The stability of these models was investigated by using an $N$-body code based on the self-consistent field method described by Hernquist \& Ostriker (1992). This scheme consists of solving the Poisson equation by expanding the density and the gravitational potential using a biortoghonal set of basis functions. For spherically symmetric systems, it is natural to

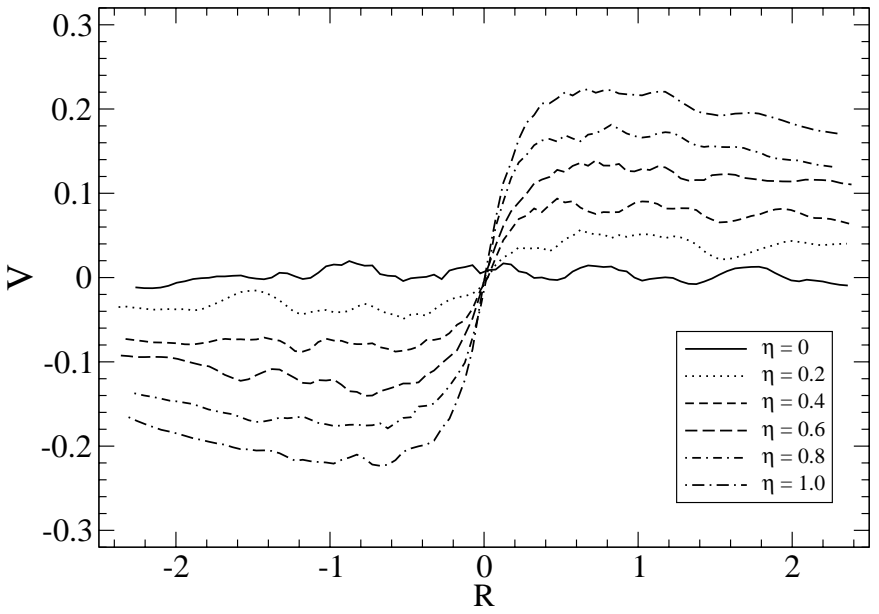

Fig. 1. Mean streaming velocity for Plummer models with different degrees of rotation $\eta$ as a function of the projected position along the $x$-axis. The systems are viewed such that the rotation axis is perpendicular to the line-of-sight.

employ spherical harmonics to expand the angular dependence. Then, the density and potential expansions become

$$
\begin{aligned}
& \rho(\boldsymbol{r})=\sum_{n l m} A_{n l m} \rho_{n l}(r) Y_{l m}(\theta, \varphi), \\
& \Phi(\boldsymbol{r})=\sum_{n l m} A_{n l m} \Phi_{n l}(r) Y_{l m}(\theta, \varphi) .
\end{aligned}
$$

In practice, these expansions are truncated at some values $n_{\max }$ and $l_{\max }$ for the radial and angular functions, respectively.

The choice of the radial basis functions $\left\{\rho_{n l}, \Phi_{n l}\right\}$ is not unique; in fact, several sets have been proposed (e.g., CluttonBrock 1973; Allen et al. 1990; Zhao 1996). However, the efficiency of this method relies upon the ability to represent the density profile of the initial system and its subsequent evolution with the first few terms of the basis set. Therefore, it is generally desirable to select a set of basis functions such that their lowest order terms provide a good approximation to the density profile of the system under study.

In these simulations, the Clutton-Brock (1973) basis set is used for the Plummer model, while the Hernquist-Ostriker (1992) basis set is employed for the other three models. The zeroth-order terms of these basis sets are, respectively, the Plummer model and the Hernquist model. The $\gamma=0$ model can be recovered by a linear combination of only two terms of the Hernquist-Ostriker basis functions (see Hernquist \& Ostriker 1992). Therefore, these basis sets provide exact representations for three of the models here studied. On the other hand, the Jaffe model can only be approximated by using a finite number of terms of the Hernquist-Ostriker basis set; in this case, good accuracy can be obtained with $\sim 10$ terms (see e.g., Meza \& Zamorano 1997).

All simulations employed $N=10^{5}$ equal-mass particles and potential expansions up to $n_{\max }=10$ for the radial functions and $l_{\max }=2$ for the angular functions. Nonzero $m$ terms were also included to allow the development of any nonaxisymmetric instabilities. Time integration was performed 

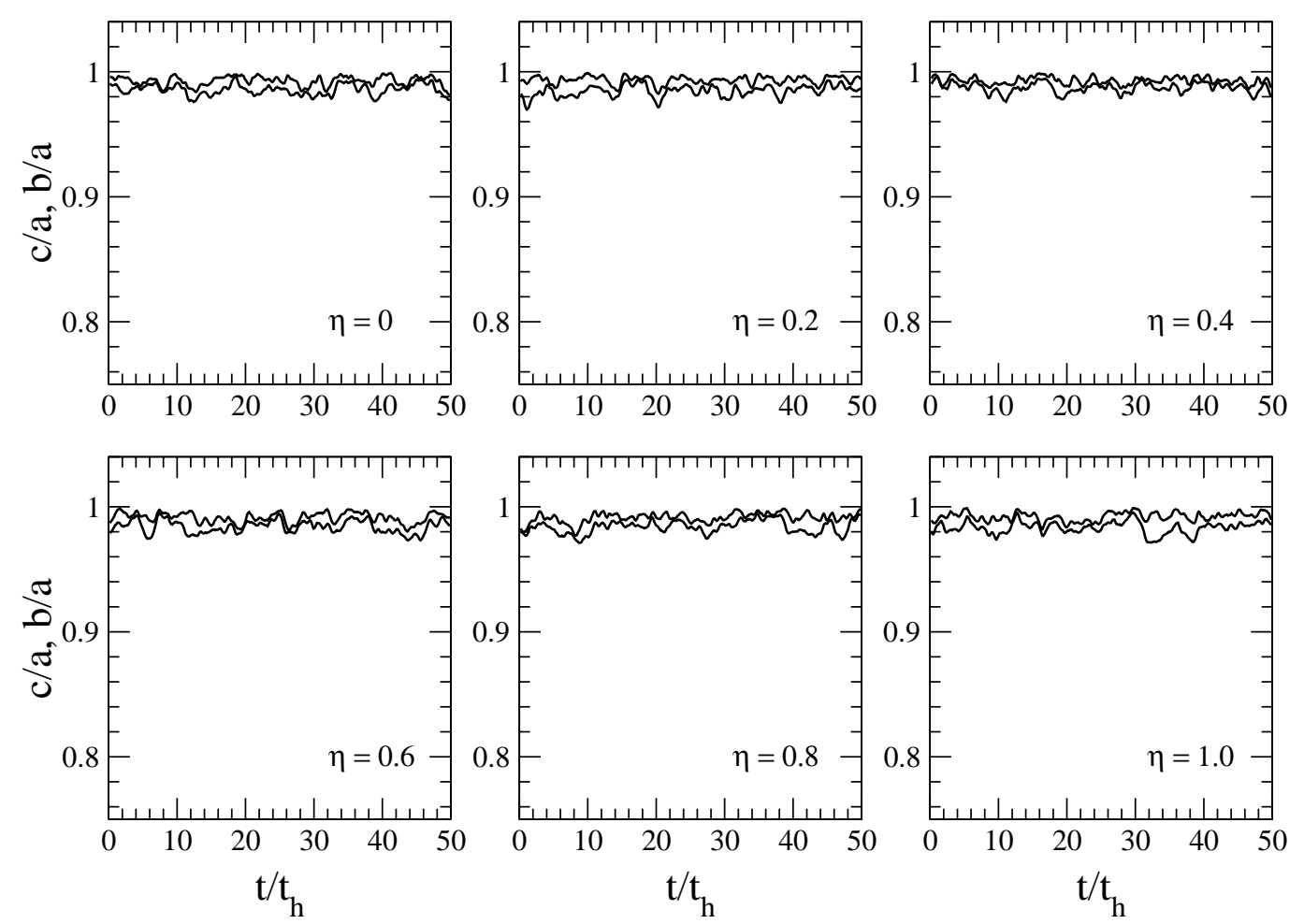

Fig. 2. Evolution of the axis ratios $c / a$ and $b / a$, with $a \geq b \geq c$, for the $\gamma=0$ models with different degrees of rotation. The axis ratios were measured at radius $r_{m}=8$, which encloses $\sim 70 \%$ of the total mass. Time has been normalized to the half-mass dynamical time, $t_{\mathrm{h}}=16.8$.

using a second order scheme with a fixed time step $\Delta t$, given by

$\boldsymbol{x}_{i+1}=\boldsymbol{x}_{i}+\Delta t \boldsymbol{v}_{i}+\frac{1}{2} \Delta t^{2} \boldsymbol{a}_{i}$

$\boldsymbol{v}_{i+1}=\boldsymbol{v}_{i}+\frac{1}{2} \Delta t\left(\boldsymbol{a}_{i}+\boldsymbol{a}_{i+1}\right)$,

where the subscript identifies the iteration (see e.g., Hut et al. 1995). The total elapsed time for all these simulations was $T_{\text {end }}=50 t_{\mathrm{h}}$, where $t_{\mathrm{h}}$ is the dynamical time evaluated at the half-mass radius of the respective model. The time step used for each model is shown in Table 1. With these parameters, the total energy was conserved to better than $0.01 \%$ for the Plummer, Hernquist and $\gamma=0$ models, while for the Jaffe models the conservation of energy was only about $4 \%$.

With the adopted value $l_{\max }=2$ for the potential expansions, these simulations are designed for searching radial $(m=0)$, lopsided $(m=1)$, and bar $(m=2)$ instabilities. A test particularly sensitive to the bar instability is the evolution of the axis ratios of the particle distribution. For each simulation, the axial ratios inside a given radius were obtained by using an iterative algorithm similar to that used by Dubinski \& Carlberg (1991). In this scheme, initial values for the inertia tensor

$I_{i j}=\sum \frac{x_{i} x_{j}}{|\boldsymbol{x}|^{2}}$

are computed for all particles inside a sphere of radius $r_{m}$. The eigenvalues and eigenvectors of $I_{i j}$ provide an approximation to the axis ratios and the orientation of the fitting ellipsoid. Then, the modified inertia tensor is evaluated only for particles inside that ellipsoid, which gives an improved approximation to their axis ratios and orientation. This process is repeated until the axis ratios converge to a value within a pre-established tolerance.

\section{Results}

A total of 24 simulations were performed. The main result of these simulations is that all the rotating models are dynamically stable. This is illustrated by displaying the evolution of some of them. The results for other models are similar.

The evolution of the axis ratios $b / a$ and $c / a$, where $a \geq$ $b \geq c$, for the $\gamma=0$ models with different degrees of rotation is shown in Fig. 2. These axis ratios were measured at radius $r_{m}=8$, which encloses $\sim 70 \%$ of the total mass. For all values of $\eta$, the axis ratios remains essentially equal to their initial values. The fluctuations reflect the discreteness of the models and are consistent with the number of particles employed in the simulations. Similar behavior is observed for the axis ratios measured at other radii.

A more sensitive test for the existence of possible instabilities is provided by the analysis of the individual expansion coefficients $A_{n l m}$, which is straightforward in the self-consistent field method because the coefficients are evaluated at each time step in order to compute the forces. The evolution of the amplitude of several expansion coefficients $A_{n l m}$ for the rotating Hernquist model with $\eta=1$ is shown in Fig. 3. These coefficients were calculated using the Hernquist \& Ostriker (1992) basis functions. As expected, the zeroth-order coefficient $A_{000} \simeq 1$, while the higher order coefficients are closer to zero and fluctuate around their initial values with amplitude consistent with the noise induced by the number of particles 


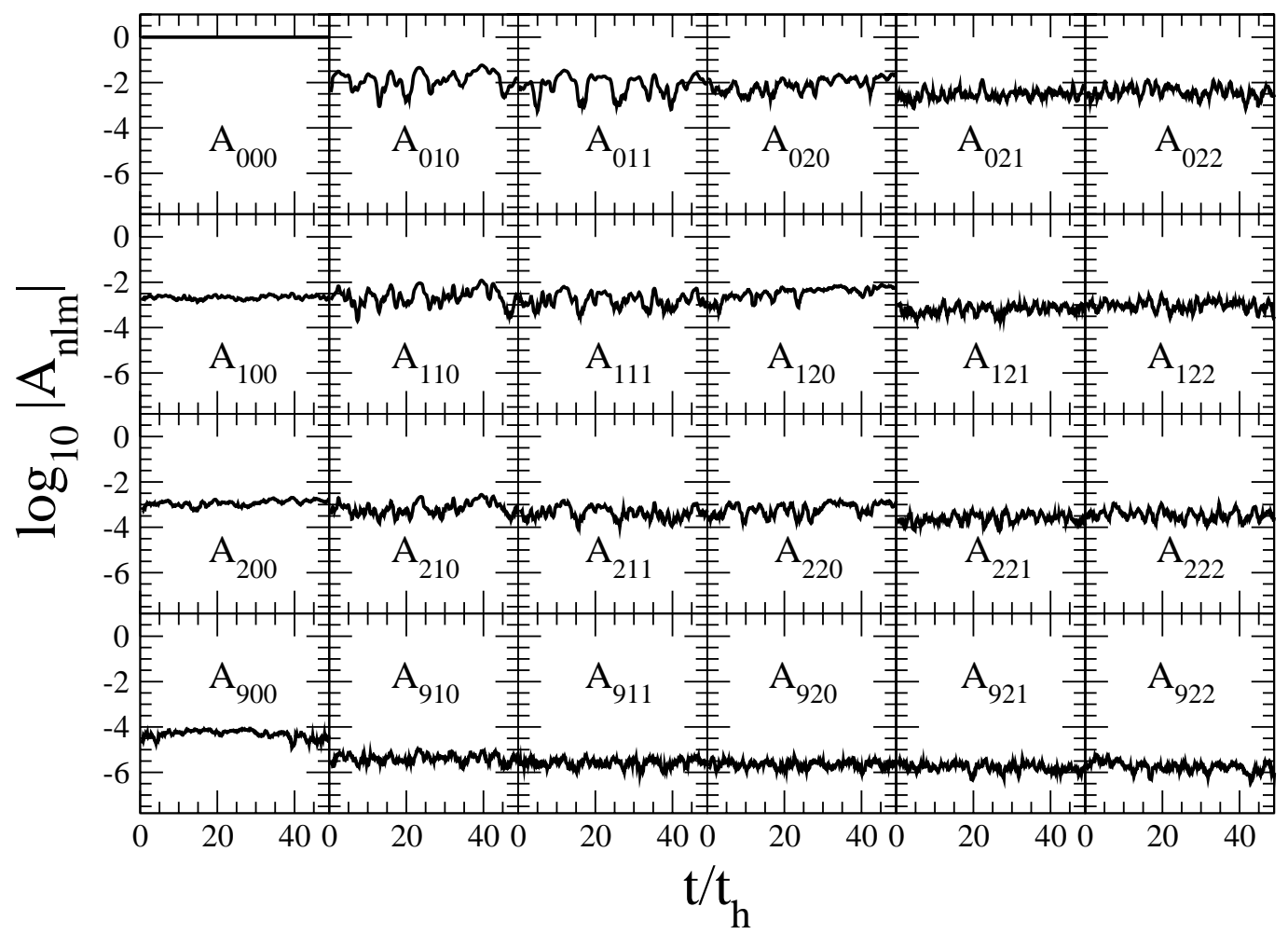

Fig. 3. Evolution of the amplitude of several expansion coefficients $A_{n l m}$ for the Hernquist model with $\eta=1$. The coefficients $A_{n l m}$ were computed using the Hernquist-Ostriker basis set, where $n$ refers to the radial functions, and $l$ and $m$ to the angular functions (spherical harmonics). The logarithm of the absolute value of the coefficients is shown. Time has been normalized to the half-mass dynamical time, $t_{\mathrm{h}}=8.33$.

used in these simulations. In particular, there are no signs of radial $(m=0)$, bar $(m=2)$, or lopsided $(m=1)$ instabilities. Other coefficients show the same qualitative behavior.

\section{Conclusions}

The stability of four rotating isotropic spherical models was investigated by using $N$-body simulations. The density profiles of these models provide good approximations to the mass distribution of globular clusters and elliptical galaxies. Different degrees of rotation were introduced in these models by reversing the sense of rotation along the $z$-axis of a given fraction of the particles (Lynden-Bell 1962). Simulations show that all these rotating models are dynamically stable, irrespective of their degree of rotation. No signs of radial, lopsided, or bar instabilities were observed. If some of them exist their growth rate is larger than $50 t_{\mathrm{h}}$, the time elapsed for these simulations.

These simulations show that spherical stellar systems can rotate very rapidly without becoming oblate. This result contrasts with the suggestion of Alimi et al. (1999) that there do not exist spherical stellar systems in fast rotation (i.e., with $\mu \gtrsim 0.1$ ). However, their conclusions were based on a series of simulations for spherical $n=4$ polytropes with isotropic velocity distributions, which were made to rotate by using a procedure that modifies their initial velocity anisotropy. Therefore, their conclusions are not strictly applicable to the isotropic models studied in this paper. But, they could still be valid for anisotropic spherical models. In fact, there are several other ways to construct rotating spherical models. For example, one could construct a system containing only circular orbits and then reverse a half of them (see Lynden-Bell 1960). Clearly, such a model would exhibit stronger rotation than the models here analyzed and surely might be unstable.

Acknowledgements. I am grateful to the referee for providing comments which improved the presentation of this paper. I thank Andreas Reisenegger and Nelson Zamorano for helpful suggestions and comments on the manuscript. I also thank the hospitality of the Department of Physics and Astronomy at the University of Victoria where the final version of this paper was written. This work was partially supported by FONDECYT grant 3990031.

\section{References}

Alimi, J., Perez, J.-M., \& Serna, A. 1999, MNRAS, 305, 859

Allen, A. J., Palmer, P. L., \& Papaloizou, J. 1990, MNRAS, 242, 576

Allen, A. J., Palmer, P. L., \& Papaloizou, J. 1992, MNRAS, 256, 695

Antonov, V. A. 1960, SvA, 4, 859

Antonov, V. A. 1962, Vestnik Lenningradskogo Univ. 19, 96, English translation in Proc. IAU Symp. 127, Structure and Dynamics of Elliptical Galaxies, ed. P. T. de Zeeuw (Reidel, Dordrecht), 531

Barnes, J., Hut, P., \& Goodman, J. 1986, ApJ, 300, 112

Binney, J., \& Tremaine, S. 1987, Galactic Dynamics (Princeton Univ. Press, Princeton)

Clutton-Brock, M. 1973, Ap\&SS, 23, 55

Dejonghe, H., \& Merritt, D. 1988, ApJ, 328, 93

Dehnen, W. 1993, MNRAS, 265, 250

Dorémus, J. P., \& Feix, M. R. 1973, A\&A, 29, 401

Dorémus, J. P., Feix, M. R., \& Baumann, G. 1971, Phys. Rev. Lett., 26,725 
Dubinski, J., \& Carlberg, R. G. 1991, ApJ, 378, 496

Hénon, M. 1973, A\&A, 24, 229

Hernquist, L. 1990, ApJ, 356, 359

Hernquist, L., \& Ostriker, J. P. 1992, ApJ, 386, 375

Hut, P., Makino, J., \& McMillan, S. 1995, ApJ, 443, L93

Jaffe, W. 1983, MNRAS, 202, 995

Kalnajs, A. J. 1977, ApJ, 212, 637

Kandrup, H. E., \& Sygnet, J. F. 1985, ApJ, 298, 27

Lynden-Bell, D. 1960, MNRAS, 120, 204

Lynden-Bell, D. 1962, MNRAS, 124, 1

Merritt, D. 1999, PASP, 111, 129

Merritt, D., \& Aguilar, L. A. 1985, MNRAS, 217, 787

Meza, A., \& Zamorano, N. 1997, ApJ, 490, 136

Miller, R. H., \& Smith, B. F. 1980, ApJ, 235, 793

Navarro, J. F., \& White, S. D. M. 1993, MNRAS, 265, 271

Palmer, P. L. 1994a, Stability of Collisionless Stellar Systems (Kluwer, Dordrecht)
Palmer, P. L. 1994b, in Proc. EADN Astrophysics School VI, Galactic Dynamics and $N$-Body Simulations, Lecture Notes Phys. 433, ed. G. Contopoulos (Springer, New York), 143

Papaloizou, J. C. B., Palmer, P. L., \& Allen, A. J. 1991, MNRAS, 253, 129

Plummer, H. C. 1911, MNRAS, 71, 460

Polyachenko, V. L. 1981, Soviet Astron. Lett., 7, 79

Press, W. H., Teukolsky, S. A., Vetterling, W. T., \& Flannery, B. P. 1992, Numerical recipes in C, The art of scientific computing (Cambridge: University Press)

Sellwood, J. A., \& Valluri, M. 1997, MNRAS, 287, 124

Spitzer, L. 1987, Dynamical Evolution of Globular Clusters (Princeton Univ. Press, Princeton)

Sygnet, J. F., Des Forets, G., Lachieze-Rey, M., \& Pellat, R. 1984, ApJ, 276, 737

Tremaine, S., Richstone, D. O., Byun, Y. I., et al. 1994, AJ, 107, 634

Zhao, H. 1996, MNRAS, 278, 488 